\title{
Pacemakers, ICDs, and MRI
}

The majority of pacemakers and implantable cardioverter-defibrillators (ICDs) currently in use are termed 'legacy' systems and do not meet the FDA-specified criteria to be labelled as safe during MRI (termed 'MRI-conditional'). Therefore, patients with legacy devices are often not allowed to undergo MRI examination. Now, data from a new study challenge this idea. "We found MRI examinations to be safe in the setting of legacy cardiac pacemakers or ICDs, when using a safety protocol," says corresponding author Saman Nazarian. "The scans were safely performed even with thoracic or cardiac MRI and in patients dependent on cardiac pacing for every heartbeat," he adds. In this prospective, nonrandomized study, the investigators performed a total of 2,103 MRI examinations at 1.5 Tesla in 1,509 patients with a legacy pacemaker (58\%) or a legacy ICD (42\%). All MRI examinations were deemed to be clinically necessary and were performed using a prespecified safety protocol. Device reset occurred during nine MRI examinations in eight patients. The reset was transient in eight examinations and the device had to be replaced in only one patient. Changes in device parameters were infrequent. Immediately after MRI, a decrease in P-wave amplitude was the most notable frequent change ( $>50 \%$ change from baseline), occurring in $1 \%$ of patients. At long-term follow-up (median 1 year), a decrease in $\mathrm{P}$-wave amplitude was again the most common change from baseline ( $4 \%$ of patients), together with increases in atrial capture threshold (4\%), right ventricular capture threshold (4\%), and left ventricular capture threshold (3\%). No long-term clinically significant adverse events were reported in any of the patients. "Given these new data, the idea that access to the potentially lifesaving diagnostic data from MRI would be limited for patients with legacy pacemakers and ICD leads or generators is outdated," concludes Nazarian.

Irene Fernández-Ruiz

ORIGINAL ARTICLE Nazarian, S. et al. Safety of magnetic resonance imaging in patients with cardiac devices. N. Engl. J.Med. 377, 2555-2564 (2017)

FURTHER READING Cingolani, E. et al. Next-generation pacemakers: from small devices to biological pacemakers. Nat. Rev. Cardiol. https://doi.org/10.1038/nrcardio.2017.165 (2017)

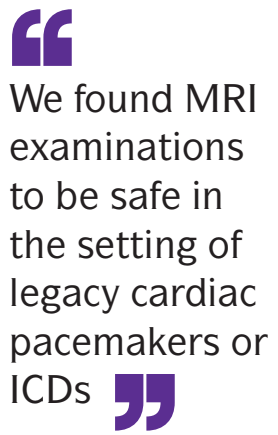

We found MRI examinations to be safe in the setting of legacy cardiac pacemakers or ICDs 